\title{
Up-regulated IncRNA-PVT1 Expression in Peripheral Blood Mononuclear Cells of Patients with Coronary Artery Disease is Correlated with Decreased Interleukin-10 Production
}

\author{
Behnoosh Miladpour \\ Fasa University of Medical Science \\ Atefeh Seghatoleslam \\ Shiraz University of Medical Sciences \\ mehdi kalani \\ Shiraz University of Medical Sciences \\ Mehran Erfani \\ Islamic Azad University of Arak \\ peyman Nowrouzi-Sohrabi ( $\square$ peynow1399@yahoo.com ) \\ Shiraz University of Medical Sciences
}

\section{Research article}

Keywords: coronary artery disease, long non-coding RNA, PVT1, interleukin-10

Posted Date: August 13th, 2020

DOI: https://doi.org/10.21203/rs.3.rs-50028/v1

License: (c) (i) This work is licensed under a Creative Commons Attribution 4.0 International License.

Read Full License 


\section{Abstract}

Background: Plasmacytoma variant translocation 1 (PVT1) is a newly discovered long non-coding RNA (IncRNA), and it has not been previously studied in the inflammatory responses of peripheral blood mononuclear cells (PBMCs) of patients with coronary artery disease (CAD).

Methods: This cross-sectional study was conducted in 15 CAD patients and 15 non-CAD (NCAD) individuals. PVT1 expression in PBMCs of the participants was measured, using real-time PCR.

Interleukin (IL)-10, IL-22 and MMP-9 in the plasma and supernatant of the cultured PBMCs in the presence or absence of lipopolysaccharide (LPS) was assessed, using flowcytometry and ELISA.

Results: An increased expression of PVT1 was observed in untreated PBMCs of CAD patients compared to the NCAD group. There was a significant up-regulation of PVT1 after LPS treatment in PBMCs of both groups. Plasma matrix metalloproteinase-9 (MMP-9) levels were found to be higher in CAD patients compared to the controls. The level of IL-10 and IL-22 production from the non-treated PBMCs of CAD was significantly lower compared to the NCAD group. In the total examined population, PVT1 expression was negatively correlated with IL-10 secretion. The results also showed a significant negative correlation between PVT1 expression and IL-10 produced by untreated cells.

Conclusions: PVT1 expression is increased in PBMCs of CAD patients and this increased expression could be associated with decreased IL-10 production from PBMCs of these patients.

\section{Background:}

Coronary artery disease (CAD) is considered as principal cause of death and disability leading to increased health economic burden worldwide (1). The most common form of CAD is atherosclerosis which is a chronic inflammatory disease of the arterial wall, arising from a maladaptive inflammatory response and an imbalance in lipid metabolism (2). Numerous converging lines of clinical and experimental evidence show that inflammation plays a pivotal role in all phases of the atherosclerotic process (3).

Previously, the role of inflammatory cytokines has been remarkably evaluated (4). It has been shown that pro-inflammatory cytokines, e.g. interleukin (IL)-1 $\beta$, and IL-6, and tumor necrosis factor alpha (TNF-a) present a pro-atherogenic effects in general, while anti-inflammatory cytokines, e.g. IL-10, could reduce the risk of atherosclerosis progression and ameliorate the development of atherosclerosis and vascular complications (5). IL-22, a member of the IL-10 related cytokine superfamily, has a dual nature in inflammation, which might present a pro-inflammatory or anti-inflammatory effect in order to modulate the tissue's immune responses (4). All in all, it seems that IL-10 and IL-22 have beneficial effects in protecting against metabolic disorders, decreasing chronic inflammation and related complications (6). 
Matrix metalloproteinases (MMPs) play a crucial role in extracellular matrix metabolism. MMPs have shown the important involvement in cardiovascular diseases, including atherosclerosis, coronary artery disease, and myocardial infarction (7). MMP-9 plays a pivotal role in the instability of atherosclerotic plaques. It can also be found in the plasma due to the proteolytic rupture of the cellular membranes. Therefore, MMP-9 can be evaluated in several clinical situations in which inflammation is an underlying contributor, e.g. CAD (8).

Long non-coding RNAs (IncRNAs), a new class of non-coding RNAs with a length of $>200$ nucleotides, are considered as a significant component in epigenetic regulation of gene expression. Increasing evidence has demonstrated that IncRNAs are involved in the regulation of numerous physiologic and pathologic processes, particularly the regulation of injury, invasion and inflammatory responses (9).

Plasmacytoma variant translocation 1 (PVT1) is a newly discovered IncRNA and is located at chromosome 8q24, a known cancer-related region. PVT1 functions as an oncogene and its up-regulation is tightly associated with a series of human cancer types (10). PVT1 dysregulation is involved in the etiology of a wide variety of human disorders such as cardiovascular diseases (11). During the past few years, PVT1 has received increasing interest due to its involvement in the aberrant inflammatory and immune responses in various diseases, including osteoarthritis $(12,13)$, epilepsy $(14)$, abdominal aortic aneurysm (15), and sepsis-induced acute kidney injury (16) and cardiac dysfunction (17). Thus, previous literature has highlighted the potential of PVT1 as a promising therapeutic target for inflammatory disorders. However, the importance of PVT1 on inflammatory responses in CAD and atherogenesis has not been thoroughly elucidated to date. Therefore, to investigate the possible role of PVT1 in immune and inflammatory responses in CAD, in the present study, we established an experimental model of lipopolysaccharide (LPS) stimulated human Peripheral blood mononuclear cells (PBMCs). Using our model, we detected the expression of PVT1 in PBMCs from patients with CAD under both LPS-stimulated and unstimulated conditions. Furthermore, the correlation of PVT1 expression with plasma levels and the ex-vivo production of IL-10 superfamily cytokines (IL-10 and IL-22), and MMP-9 by PBMCs was investigated.

\section{Methods}

\section{Study design and participants}

This study was approved by medical ethics committee of Fasa University of Medical Sciences and an informed written consent was obtained from all the participants. Our study was performed in accordance with the principles of the Declaration of Helsinki (18). Thirty peripheral blood samples were collected from male patients who had undergone $\mathrm{CT}$ angiography or coronary angiography for suspected coronary artery disease in the Al Zahra Heart Hospital (Shiraz, Iran) between October 2018 and January 2019. We divided the patients into two groups including CAD and non-CAD (NCAD), each containing 15 patients. The diagnosis of CAD was made by a cardiologist if any patients had more than $50 \%$ stenosis in at least one coronary artery proven by angiography. Exclusion criteria were diabetes mellitus, malignancy, 
infection, blood diseases and chronic renal or liver failure, history of inflammatory diseases, and use of immunosuppressive drugs.

\section{Isolation and culture of PBMCs}

To prepare the PBMCs, $10 \mathrm{ml}$ peripheral blood samples drawn from participants were collected in in sterile tubes with EDTA. Centrifugation was done over Ficoll-Hypaque gradients (Lymphodex, InnoTrain, Germany) at $400 \mathrm{~g}$ for $20 \mathrm{~min}$ at $4^{\circ} \mathrm{C}$. The interface fraction containing PBMCs was carefully collected and washed twice with RPMI 1640 medium. The PBMCs were cultured in the RPMI medium supplemented with $50 \mathrm{U} / \mathrm{mL}$ penicillin, $50 \mu \mathrm{g} / \mathrm{mL}$ streptomycin and $10 \%$ fetal bovine serum at $37^{\circ} \mathrm{C}$ in $5 \%$ CO2 incubator. In order to evaluate the PVT1 expression, $2 \times 10^{6} \mathrm{PBMCs} /$ well were incubated in the presence or absence of $100 \mathrm{ng} / \mathrm{mL}$ of LPS (Sigma-Aldrich, Germany) for 5 hours in 24-well culture plates. Also, $10^{5}$ PBMCs/well were cultured in the presence or absence of $100 \mathrm{ng} / \mathrm{mL}$ LPS with 48 hours of incubation in 96-well culture plates in order to evaluate the amounts of IL-10, IL-22, and MMP-9 in cell culture supernatants

\section{RNA isolation and quantitative real-time reverse transcription PCR (qRT-PCR)}

The total RNA from PBMCs cell cultures was extracted using the One Step-RNA Reagent (Bio Basic, Germany), according to its manufacturer's protocol. First-strand cDNA was synthesized using the RevertAid First Strand cDNA Synthesis Kit (Thermo Scientific, USA). The qRT-PCR for PVT1 beta-actin as the internal reference gene was carried out, using the $A B I 7500$ Sequence Detection System (Applied Biosystems, USA). The primer sequences were as follows: PVT1: Sense 5'-GTCTTGGTGCTCTGTGTT-3', anti-sense 5'-CCCGTTATTCTGTCCTTCTC-3'; Beta-actin: Sense 5'-GCCTTTGCCGATCCGC-3', anti-sense 5'GCCGTAGCCGTTGTCG-3'. The $2^{-\triangle C T}$ method was used to calculate the relative gene expression.

\section{MMP-9 measurements}

MMP-9 in the plasma and PBMCs cell culture supernatants was detected, using MMP-9 ELISA kit from BT $L A B$ (BT LAB, China) according to the manufacturer's instructions.

\section{Cytokines assay}

The plasma samples and the supernatants of the cultured PBMCs were used to assess IL-10 and IL-22 levels. Cytokines were measured by LEGENDplex ${ }^{\text {TM }}$ Human Th22 Panel (Biolegend, USA), using the flowcytometer (FACS Calibur, BD, USA), according to the manufacture's instruction.

\section{Statistical analysis}

The SPSS 22.0 (IBM Inc., USA) and GraphPad Prism version 8.2 (San Diego, CA, USA) were used to perform statistical analysis. The normality of the continuous variables was evaluated, using KolmogorovSmirnov test. Normal variables are presented as mean and standard deviation (SD) and non-parametric variables are presented by median and interquartile range (IQR). The study groups were compared using 
Student's T test, Mann-Whitney U test or one-way ANOVA. The Spearman correlation test was used to determine the relationship of PVT1 expression with other variables. $\mathrm{P}<0.05$ was regarded statistically significant.

\section{Results}

\section{Characteristics of the study population}

The demographic and anthropometric characteristics of the study population are as presented in Table 1. There were no significant differences in age, waist circumference (WC), hip circumference (HC), body mass index (BMI), and weight to hip ratio (WHR) between the study groups.

Table 1. The demographic and clinical characteristics of the study groups

\begin{tabular}{|llll|}
\hline Variable & NCAD $(n=15)$ & CAD $(n=15)$ & P value \\
\hline Age (years) & $56.20(5.82)$ & $58.87(5.76)$ & 0.217 \\
\hline WC $(\mathrm{cm})$ & $94.0(89.0-97.0)$ & $93.0(86.0-98.0)$ & 0.653 \\
HC $(\mathrm{cm})$ & $85.67(7.49)$ & $80.80(6.48)$ & 0.067 \\
BMI $\left(\mathrm{kg} / \mathrm{m}^{2}\right)$ & $26.60(2.31)$ & $25.59(3.34)$ & 0.344 \\
WHR & $0.91(0.12)$ & $0.86(0.11)$ & 0.240
\end{tabular}

CAD: coronary artery disease, NCAD: non-CAD, WC: waist circumference, HC: hip circumference, BMI: body mass index, WHR: weight to hip ratio.

\section{PVT1 expression in PBMCs}

The relative expression of PVT1 were first assessed at the basal stage in PBMCs of NCAD and CAD groups. The relative expression of PVT1 was significantly higher in CAD patients compared to the NCAD group $(p=0.034)$ (Fig 1). In addition, PVT1 expression in PBMCs of NCAD $(p=0.002)$ and CAD $(p=$ 0.001 ) increased significantly upon stimulation.

Fig 1. PVT1 expression in the PBMCs of patients with CAD and NCAD. PVT1 relative expression in PBMCs of the CAD and NCAD patients in the absence and presence of LPS $(100 \mathrm{ng} / \mathrm{mL})$ for $5 \mathrm{~h}$ was measured, using real-time PCR. *P value $<0.05 ; * \star P$ value $<0.01$

\section{Plasma levels of MMP-9, IL-10 and IL-22}

Plasma levels of MMP-9 and cytokines IL-10 and IL-22 were measured to determine the inflammatory conditions as well as their correlation with PVT1 expressions. As presented in Fig. 2, only MMP-9 was significantly $(P=0.037)$ higher in CAD patients compared to the controls, and the levels of cytokines were comparable between the investigated groups. 
Fig 2. Comparison of MMP-9, IL-10 and IL-22 in the plasma of NCAD and CAD patients. (A) MMP-9 was significantly higher in CAD patients. (B and C) IL-10 and IL-22 was comparable between NCAD and CAD patients. * $P$ value $<0.05$

\section{MMP-9, IL-10 and IL-22 production by PBMCs}

In order to assess the effect of LPS and correlation of PVT1 with MMP-9 and anti-inflammatory cytokines production, we also analyzed the supernatant of PBMCs in the absence and presence of LPS after $48 \mathrm{~h}$. The level of IL-10 production was significantly lower in the non-treated PBMCs of CAD $(p=0.002)$ and LPS-induced PBMCs of the NCAD group $(p=0.048)$ compared to non-treated PBMCs of the NCAD group (Fig 3B). The secretion of IL-22 was also significantly lower in the untreated PBMCs of the CAD group compared to the NCAD group ( $p=0.044)$ (Fig 3C).

Figure 3. Alterations in MMP-9, IL-10 and IL-22 production, following stimulation of the isolated PBMCs with LPS. Isolated PBMCs were cultured in the presence or absence of LPS $(100 \mathrm{ng} / \mathrm{mL})$ for $48 \mathrm{~h}$. Then, concentrations of MMP-9, IL-10 and IL-22 were measured in the culture supernatants. * $\mathrm{P}$ value $<0.05$ and $* * P$ value $<0.01$

\section{Association of PVT1 with MMP-9, IL-10 and IL-22}

Correlation analyses were performed to assess the association of PVT1 expression with MMP-9, IL-10 and IL-22 cytokines expression in PBMCs (Table 2). A significant negative correlation was observed between PVT1 expression and supernatant IL-10 in the supernatant of untreated PBMCs $(r=-0.399, p=$ 0.043).

\begin{tabular}{|lll|}
\hline \multicolumn{2}{|l|}{ Table 2. The correlations between PVT1 expressions with other variables } \\
\hline Variables & Untreated & LPS-treated \\
\hline Plasma MMP-9 $(\mathrm{pg} / \mathrm{ml})$ & 0.220 & -0.023 \\
\hline Plasma IL-10 $(\mathrm{pg} / \mathrm{ml})$ & 0.008 & 0.089 \\
\hline Plasma IL-22 $(\mathrm{pg} / \mathrm{ml})$ & -0.138 & 0.034 \\
\hline Supernatant MMP-9 $(\mathrm{pg} / \mathrm{ml})$ & 0.307 & 0.186 \\
\hline Supernatant IL-10 $(\mu \mathrm{g} / \mathrm{ml})$ & $-0.399 *$ & -0.345 \\
\hline Supernatant IL-22 $(\mu \mathrm{g} / \mathrm{ml})$ & -0.361 & -0.528 \\
\hline $\begin{array}{l}\text { MMP: Matrix metallopeptidase, IL: Interleukin. The Bold value is statistically significant. * P value }< \\
0.05\end{array}$ & \\
\hline
\end{tabular}

\section{Discussion}


Our data demonstrated that PVT1 was significantly up-regulated in the PBMCs of the CAD patients. Up- or down-regulated expression of CAD has been reported from different pathological states. It was reported that PVT1 expression is decreased in the dorsal root ganglia of diabetic rats (17). In addition, Wang et al. reported that down-regulated long non-coding RNA PVT1 contributes to human gestational diabetes mellitus and preeclampsia and the PVT1 expression was lower in the gestational diabetes mellitus and preeclampsia placentas than the normal placentas (19). Another study reported that PVT1 was downregulated in clinical villi samples from spontaneous abortion (20). In contrast to these findings, an increased expression of PVT1 has also been previously reported. The level of PVT1 expression was significantly increased in cancers. A meta-analysis on a total of 23 studies reported that high PVT1 expression levels correlated with poor overall survival, larger tumor size, distant metastasis, and some other risk factors in cancer patients (21). In addition, it is found to be up-regulated in atrial muscle tissues from atrial fibrillation patients (22), and the myocardial tissues of sepsis rats (23). More importantly, a very recent study by Quan et al. reported a similar finding to our findings, in which PVT1 was highly expressed in the serum of CAD patients (24). Furthermore, it was demonstrated that PVT1 directly correlated with Gensini score in CAD patients, suggesting the involvement of PVT1 in the development of atherosclerosis. In this paper, PVT1 was highly expressed in PBMCs of CAD patients compared to healthy controls, and also up-regulated upon LPS-stimulation in both groups.

To study the role of PVT1 in the production of the IL-10 super family cytokines and MMP-9, we assessed the IL-10, IL-22, and MMP-9 production in both basal and LPS treated states. IL-10 is a pleiotropic cytokine with immune-regulatory properties, produced mostly by Tregs and B regulatory cells. Due to its anti-inflammatory properties, IL-10 is believed to have inhibitory effects on the plaque development and atherosclerosis progression (25). In the present study, we also found that as PVT1 increases, the production of IL-10 is reduced from PBMCs of the total population examined. Therefore, PVT1 might be the factor contributing to anti-inflammatory cytokine IL-10 secretion from PBMCs.

To the best of our knowledge, this is the first to show the relationship between PVT1 expression and IL-10 production in NCAD and CAD patients. Zhao et al. reported high expression of PVT1 in the cartilage of osteoarthritis patients and IL-1 $\beta$-stimulated chondrocytes. Moreover, in support of this finding, it has been reported that a defect cessation of PVT1 inhibition antagonized the production of inflammatory cytokines upon IL-1 $\beta$ stimulation, including prostaglandin E2, IL-6, IL-8 and TNF- $a$. However, the precise molecular mechanisms of this correlation is not well understood; however, different factors might be involved, such as anti-inflammatory microRNAs, e.g. microRNA (miR)-146a, miR-27b-3.

Several studies have reported the association between miR-14a and CAD; the results of a recent systematic review and meta-analysis supported the existence of a role for miR-146a rs2910164 polymorphism in determining the susceptibility to cardio-cerebrovascular diseases, especially CAD (26). Another study reported that the expression of miR-146a in PBMCs and plasma was significantly higher in CAD patients compared to NCAD patients (27). In addition, it has been suggested that PVT1 is a mediator of miR-146a expression by inducing the methylation of $\mathrm{CpG}$ Island in its promoter so that the miR-146a overexpression eliminates the effects of PVT1 knockdown on prostate cancer cells. Liu et al. showed that 
miR-146a was down-regulated and negatively correlated with the PVT1 level in prostate cancer. PVT1 mediated miR-146a expression by inducing the methylation of $\mathrm{CpG}$ Island in its promoter. MiR-146a overexpression eliminated the effects of PVT1 knockdown on prostate cancer cells (28). It should be noted that miR-146a, as a target of PVT1, has been documented as an anti-inflammatory mediator (29), which not only correlates with increased production of anti-inflammatory cytokines TGF- $\beta 1$ and IL-10 (30), but also distinguishes CAD patients from NCAD patients, as mentioned above. MiR-27b-3p could be another possible mediator to justify the observed correlation between PVT1 and IL-10. Complementary sequences of PVT1 and miR-27b-3p has been predicted. In addition, the effect of PVT1 on miR-27b-3p has been previously evaluated in C28/I2 human chondrocyte cell line, and results showed that overexpression of PVT1 significantly decreased, and interference of PVT1 increased the miR-27b-3p expression (13). Rouas et al. showed that overexpression of miR-27b-3p, down-regulated IL-10 expression upon targeting its 3'UTR in T-cells; however, to the best of our knowledge, the deregulation of miR-27b-3p in CAD patients has not been reported yet (31).

In this study, we also found a higher level of MMP-9 in the plasma of CAD patients compared to healthy controls. However, in spite of some other studies in other pathologic conditions, e.g. in aortic aneurysm model (12), and lung cancer cell lines (23), no significant correlation was observed between PVT1 expression and plasma or supernatant levels of MMP-9.

So far, there has been relatively little research conducted on the relationship between IL-22 and coronary artery disease. According to the study of Gong et al., the elevated serum IL-22 was associated with the incidence of type 2 diabetes mellitus and CAD. Furthermore, they showed IL-22 was able to protect the endothelial cells against lysophosphatidylcholine high and glucose-induced injury (32). In the present study, our results demonstrated that the serum IL-22 level elevated in response to the LPS-induced inflammation. Therefore, it could be suggested that keeping IL-22 levels high, through inhibiting inflammation, can be another therapeutic therapeutic target for microvascular diseases with chronic lowgrade inflammation, such as diabetes and CAD. However, interleukin has been introduced as a doubleedged sword (32), and more studies are needed in this area.

\section{Conclusion}

PVT1 expression is increased in the PBMCs of CAD patients and this increased expression could be associated with decreased IL-10 production from PBMCs of these patients. However, further in-vivo and in-vitro studies are required to explore the precise molecular mechanism of PVT1 up-regulation and its association with inflammatory responses in CAD patients.

\section{Abbreviations}

Coronary artery disease

CAD

tumor necrosis factor alpha 
TNF-a

Long non-coding RNAs

IncRNAs

Matrix metalloproteinases

MMPs

Plasmacytoma variant translocation 1

PVT1

Peripheral blood mononuclear cells

PBMCs

\section{Declarations}

Ethics approval and consent to participate: Prior to data collection, all respondents were informed about the aims of the study and data confidentiality, and gave written informed consent. All participants took part in the survey voluntarily. ethic No. IR.FUMS.REC.1397.144.

Consent for publication: not applicable.

Availability of data and materials: The datasets used and/or analysed during the current study are available from the corresponding author on reasonable request.

Competing interests: The authors have declared that no conflict of interest exists (financial, non-financial) and they have consent to the publication.

Funding: This study was funded by Fasa University of Medical Sciences, NO. 97093. Behnoosh Miladpour (who is funded) had several roles that are describe at "Authors' contributions" section.

Authors' contributions: Conceptualization: BM, PNS methodology: PNS, MK, ME, data curation and analysis: BM, PNS, AS, writing-original draft preparation: PNS, writing-review and editing: BM, AS, PNS, the authors read and approved the final manuscript.

Acknowledgements: We greatly appreciate Fasa university of medical sciences for funding this project. We also thank the assistance provided by the staff of the Al-Zahra Heart Hospital of Shiraz, Iran.

ethics committee: ethic No. IR.FUMS.REC.1397.144

\section{References}

1. Libby P, Loscalzo J, Ridker PM, Farkouh ME, Hsue PY, Fuster V, et al. Inflammation, immunity, and infection in atherothrombosis: JACC review topic of the week. J Am Coll Cardiol. 2018;72(17):207181.

2. Silvestre-Roig C, de Winther MP, Weber C, Daemen MJ, Lutgens E, Soehnlein O. Atherosclerotic plaque destabilization: mechanisms, models, and therapeutic strategies. Circulation research. 
2014;114(1):214-26.

3. Nguyen MT, Fernando S, Schwarz N, Tan J, Bursill CA, Psaltis PJ. Inflammation as a therapeutic target in atherosclerosis. Journal of clinical medicine. 2019;8(8):1109.

4. Akbari H, Asadikaram G, Jafari A, Nazari-Robati M, Ebrahimi G, Ebrahimi N, et al. Atorvastatin, Iosartan and captopril may upregulate IL-22 in hypertension and coronary artery disease; the role of gene polymorphism. Life Sci. 2018;207:525-31.

5. Zhu Z, Zhang Y, Ye J, Wang X, Fu X, Yin Y, et al. IL-35 promoted STAT3 phosphorylation and IL-10 production in $B$ cells, but its production was reduced in patients with coronary artery diseases. Hum Immunol. 2018;79(12):869-75.

6. Wang X, Ota N, Manzanillo P, Kates L, Zavala-Solorio J, Eidenschenk C, et al. Interleukin-22 alleviates metabolic disorders and restores mucosal immunity in diabetes. Nature. 2014;514(7521):237-41.

7. Tokuhara CK, Santesso MR, Oliveira GSNd V, Doyama TMdS, Zambuzzi JT. WF, et al. Updating the role of matrix metalloproteinases in mineralized tissue and related diseases. Journal of Applied Oral Science. 2019;27.

8. Santos JCD, Cruz MS, Bortolin RH, Oliveira KM, Araújo JNG, Duarte VHR, et al. Relationship between circulating VCAM-1, ICAM-1, E-selectin and MMP9 and the extent of coronary lesions. Clinics (Sao Paulo, Brazil). 2018;73:e203.

9. Chen X, Tang FR, Arfuso F, Cai WQ, Ma Z, Yang J, et al. The Emerging Role of Long Non-Coding RNAs in the Metastasis of Hepatocellular Carcinoma. Biomolecules. 2019;10(1).

10. Wang W, Zhou R, Wu Y, Liu Y, Su W, Xiong W, et al. PVT1 promotes cancer progression via microRNAs. Frontiers in oncology. 2019;9.

11. Wu Z-y, Trenner M, Boon RA, Spin JM, Maegdefessel L. Long noncoding RNAs in key cellular processes involved in aortic aneurysms. Atherosclerosis. 2019.

12. Zhao Y, Zhao J, Guo X, She J, Liu Y. Long non-coding RNA PVT1, a molecular sponge for miR-149, contributes aberrant metabolic dysfunction and inflammation in IL-1 $\beta$-simulated osteoarthritic chondrocytes. Bioscience reports. 2018;38(5).

13. Lu X, Yu Y, Yin F, Yang C, Li B, Lin J, et al. Knockdown of PVT1 inhibits IL-1 $\beta$-induced injury in chondrocytes by regulating miR-27b-3p/TRAF3 axis. Int Immunopharmacol. 2020;79:106052.

14. Zhao T, Ding Y, Li M, Zhou C, Lin W. Silencing IncRNA PVT1 inhibits activation of astrocytes and increases BDNF expression in hippocampus tissues of rats with epilepsy by downregulating the Wnt signaling pathway. Journal of cellular physiology. 2019;234(9):16054-67.

15. Zhang Z, Zou G, Chen X, Lu W, Liu J, Zhai S, et al. Knockdown of IncRNA PVT1 inhibits vascular smooth muscle cell apoptosis and extracellular matrix disruption in a murine abdominal aortic aneurysm model. Mol Cells. 2019;42(3):218.

16. Huang W, Lan X, Li X, Wang D, Sun Y, Wang Q, et al. Long non-coding RNA PVT1 promote LPSinduced septic acute kidney injury by regulating TNFa and JNK/NF-KB pathways in HK-2 cells. Int Immunopharmacol. 2017;47:134-40. 
17. Feng F, Qi Y, Dong C, Yang C. PVT1 regulates inflammation and cardiac function via the MAPK/NF-KB pathway in a sepsis model. Exp Ther Med. 2018;16(6):4471-8.

18. World Medical Association Declaration. of Helsinki: ethical principles for medical research involving human subjects. Jama. 2013;310(20):2191-4.

19. Wang Q, Lu X, Li C, Zhang W, Lv Y, Wang L, et al. Down-regulated long non-coding RNA PVT1 contributes to gestational diabetes mellitus and preeclampsia via regulation of human trophoblast cells. Biomedicine \& pharmacotherapy = Biomedecine \& pharmacotherapie. 2019;120:109501.

20. Qin X, Chen Y, Chen S, Liu X, Zeng W, Tian F, et al. Plasmacytoma variant translocation 1 (PVT1) regulates trophoblast viability, proliferation, and migration and is downregulated in spontaneous abortion. American journal of reproductive immunology (New York, NY: 1989). 2019;81(1):e13048.

21. Ma C, Nie XG, Wang YL, Wu DP, Liang QD. Meta-analysis of the prognostic value of long non-coding RNA PVT1 for cancer patients. Medicine. 2018;97(49):e13548.

22. Cao F, Li Z, Ding WM, Yan L, Zhao QY. LncRNA PVT1 regulates atrial fibrosis via miR-128-3p-SP1TGF- $\beta 1-S m a d$ axis in atrial fibrillation. Molecular medicine (Cambridge Mass). 2019;25(1):7.

23. Zhang Z, Zou G, Chen X, Lu W, Liu J, Zhai S, et al. Knockdown of IncRNA PVT1 Inhibits Vascular Smooth Muscle Cell Apoptosis and Extracellular Matrix Disruption in a Murine Abdominal Aortic Aneurysm Model. Mol Cells. 2019;42(3):218-27.

24. Quan W, Hu PF, Zhao X, Lianhua CG, Batu BR. Expression level of IncRNA PVT1 in serum of patients with coronary atherosclerosis disease and its clinical significance. Eur Rev Med Pharmacol Sci. 2020;24(11):6333-7.

25. Jackowska P, Chałubiński M, Łuczak E, Wojdan K, Gorzelak-Pabis P, Olszewska-Banaszczyk M, et al. The influence of statin monotherapy and statin-ezetimibe combined therapy on FoxP3 and IL 10 mRNA expression in patients with coronary artery disease. Advances in clinical and experimental medicine: official organ. Wroclaw Medical University. 2019;28(9):1243-8.

26. Bastami M, Choupani J, Saadatian Z, Zununi Vahed S, Mansoori Y, Daraei A, et al. miRNA Polymorphisms and Risk of Cardio-Cerebrovascular Diseases: A Systematic Review and MetaAnalysis. International journal of molecular sciences. 2019;20(2).

27. Zhu G-f, Chu T, Ruan Z, Zhang M, Zhou M, Zhang Q, et al. Inflammation-Related MicroRNAs Are Associated with Plaque Stability Calculated by IVUS in Coronary Heart Disease Patients. Journal of Interventional Cardiology. 2019;2019:9723129.

28. Liu HT, Fang L, Cheng YX, Sun Q. LncRNA PVT1 regulates prostate cancer cell growth by inducing the methylation of miR-146a. Cancer medicine. 2016;5(12):3512-9.

29. Wang Y, Lyu X, Wu X, Yu L, Hu K. Long non-coding RNA PVT1, a novel biomarker for chronic obstructive pulmonary disease progression surveillance and acute exacerbation prediction potentially through interaction with microRNA-146a. Journal of clinical laboratory analysis. 2020:e23346.

30. Tang H, Lai Y, Zheng J, Chen K, Jiang H, Xu G. MiR-146a Promotes Tolerogenic Properties of Dendritic Cells and through Targeting Notch1 Signaling. Immunol Investig. 2020;49(5):555-70. 
31. Rouas R, Merimi M, Najar M, El Zein N, Fayyad-Kazan M, Berehab M, et al. Human CD8(+) CD25 (+) CD127 (low) regulatory T cells: microRNA signature and impact on TGF- $\beta$ and IL-10 expression. $J$ Cell Physiol. 2019;234(10):17459-72.

32. Gong F, Wu J, Zhou P, Zhang M, Liu J, Liu Y, et al. Interleukin-22 Might Act as a Double-Edged Sword in Type 2 Diabetes and Coronary Artery Disease. Mediat Inflamm. 2016;2016:8254797.

\section{Figures}

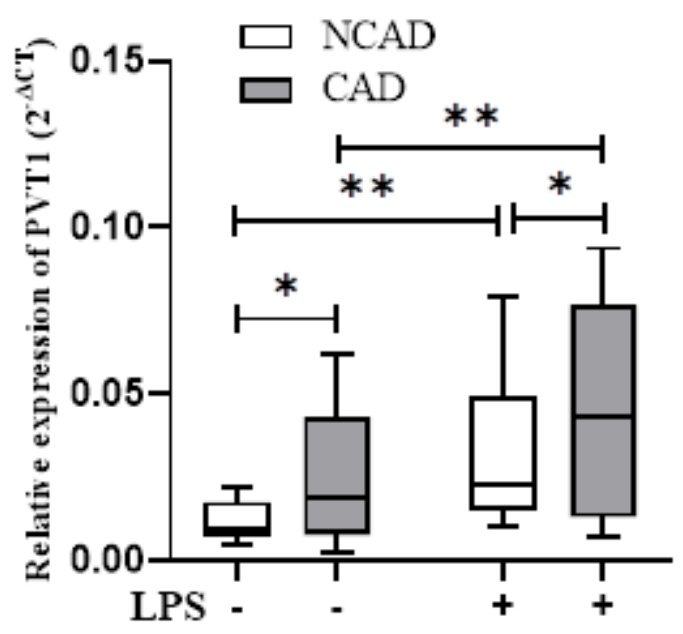

\section{Figure 1}

PVT1 expression in the PBMCs of patients with CAD and NCAD. PVT1 relative expression in PBMCs of the CAD and NCAD patients in the absence and presence of LPS $(100 \mathrm{ng} / \mathrm{mL})$ for $5 \mathrm{~h}$ was measured, using real-time PCR. *P value $<0.05 ; * * P$ value $<0.01$
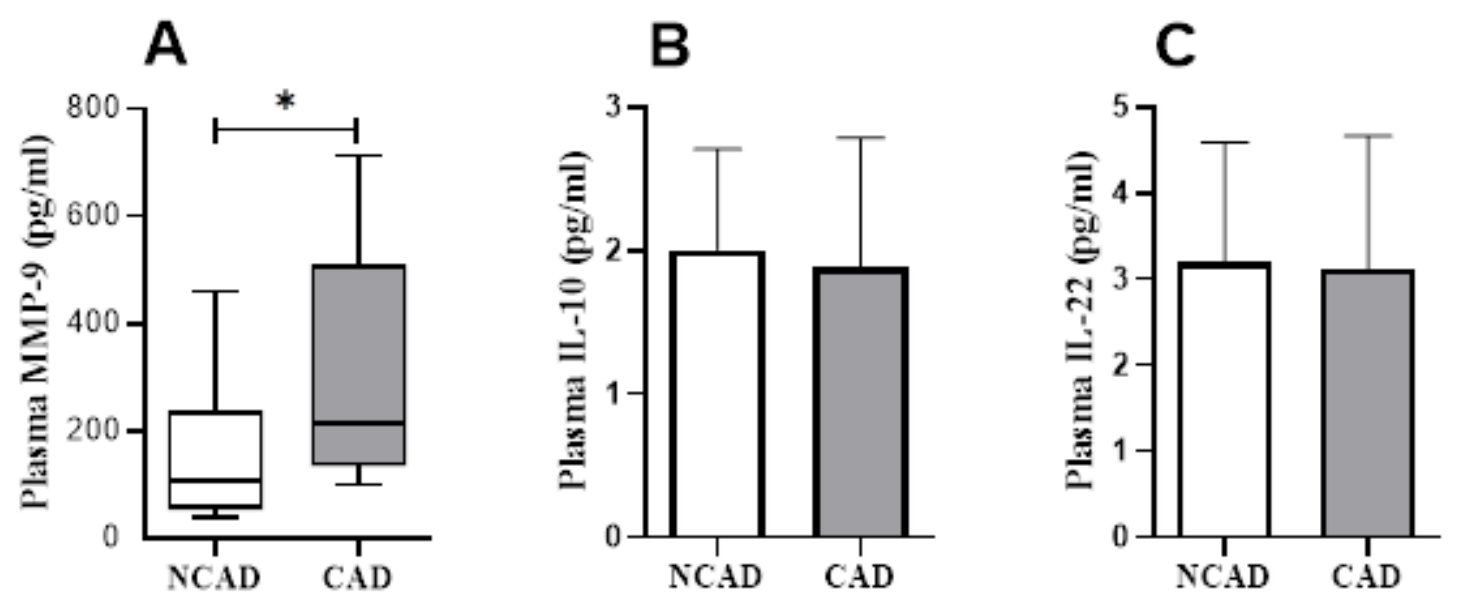

Figure 2 
Comparison of MMP-9, IL-10 and IL-22 in the plasma of NCAD and CAD patients. (A) MMP-9 was significantly higher in CAD patients. (B and C) IL-10 and IL-22 was comparable between NCAD and CAD patients. * P value $<0.05$
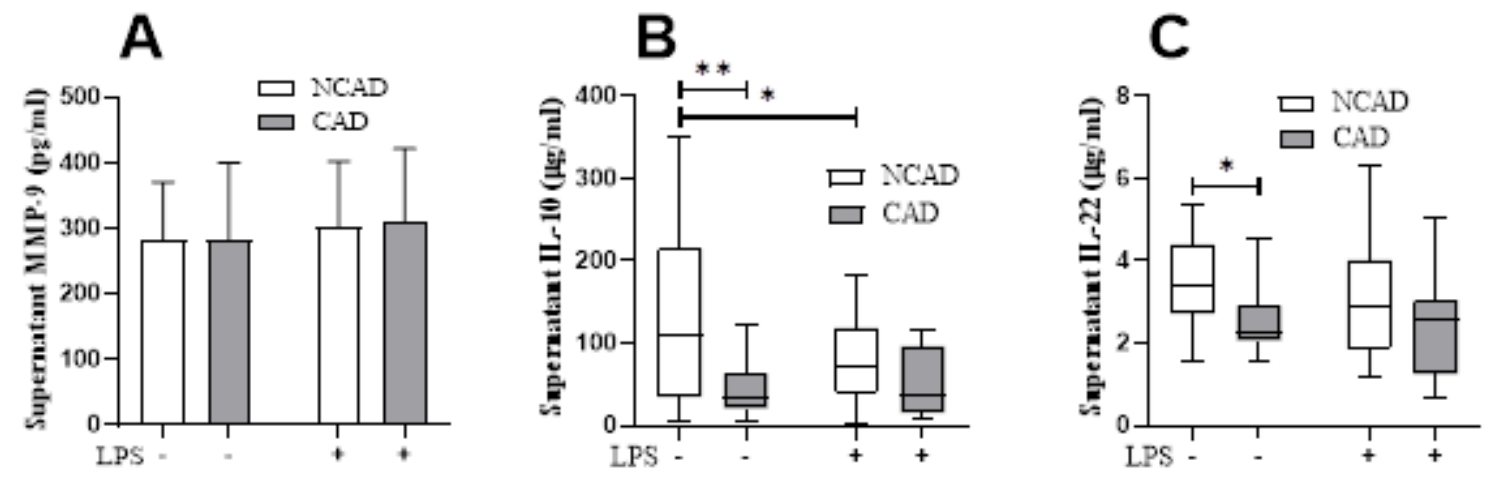

Figure 3

Alterations in MMP-9, IL-10 and IL-22 production, following stimulation of the isolated PBMCs with LPS. Isolated PBMCs were cultured in the presence or absence of LPS $(100 \mathrm{ng} / \mathrm{mL})$ for $48 \mathrm{~h}$. Then, concentrations of MMP-9, IL-10 and IL-22 were measured in the culture supernatants. * $\mathrm{P}$ value $<0.05$ and $* *$ value $<0.01$

\section{Supplementary Files}

This is a list of supplementary files associated with this preprint. Click to download.

- figures.docx 